\title{
Deuteration effects in the thermal conductivity of molecular glasses
}

\author{
A.I. Krivchikov ${ }^{1}$, F.J. Bermejo ${ }^{2}$, I.V. Sharapova ${ }^{1}$, O.A. Korolyuk ${ }^{1}$, and O.O. Romantsova ${ }^{1}$ \\ ${ }^{1}$ B. Verkin Institute for Low Temperature Physics and Engineering of the National Academy of Sciences of Ukraine \\ 47 Lenin Ave, Kharkov 61103, Ukraine \\ ${ }^{2}$ Instituto de Estructura de la Materia, CSIC, and Dept. Electricidad y Electrónica-Unidad Asociada CSIC, Facultad \\ de Ciencia y Tecnología, Universidad del País Vasco/EHU, P.O. Box 644, E-48080-Bilbao, Spain \\ E-mail: krivchikov@ilt.kharkov.ua
}

Received December 17, 2010

\begin{abstract}
The thermal conductivity $\kappa(T)$ of pure deuterated ethanol has been measured under its equilibrium vapor pressure in its orientationally-ordered crystal $\left(T=2 \mathrm{~K}-T_{m}\right)$, orientational glass and the glass state $\left(T=2 \mathrm{~K}-T_{g}, T_{g}\right.$ is the glass transition temperature) solid phases. The temperature dependence of the conductivity is well described by a sum of two contributions: $\kappa(T)=\kappa_{\mathrm{I}}(T)+\kappa_{\mathrm{II}}(T)$, where $\kappa_{\mathrm{I}}(T)$ account for the heat transport by acoustic phonons and $\kappa_{\mathrm{II}}(T)$ for the heat transfer by localized high-frequency excitations respectively. The thermal conductivities of deuterated and hydrogenated ethanols are compared in different phases. The phonon scattering mechanisms in the glasses have been analyzed. In the investigated glasses the effect of complete deuteration shows up as a contribution $\kappa_{\mathrm{II}}(T)$.
\end{abstract}

PACS: 66.70.-f Nonelectronic thermal conduction and heat-pulse propagation in solids; thermal waves;

63.20.-e Phonons in crystal lattices;

63.50.- $\mathbf{x}$ Vibrational states in disordered systems.

Keywords: thermal conductivity, crystals, glasses, deuterated ethanol, simple monoatomic alcohols, phonon scattering, disordering, soft potential model, Boson peak.

\section{Introduction}

At low temperatures the properties of disordered solids are drastically different from those of fully ordered crystals. The anomalous properties observed in various types of glasses (ionic, covalent, molecular) have much in common and are weakly dependent on the particular material structure or chemical composition. The thermal conductivity $\kappa(T)$ of a glass exhibits a universal behavior. As the temperature rises, the thermal conductivity increases as $\kappa(T) \sim T^{2}$, reaches a "plateau" at $T=5-30 \mathrm{~K}$ and then continues to increase by the quasilinear law. The glassy behavior of $\kappa(T)$ is also typical of crystals with various types of disorder (glassy crystals, clathrate compounds, ferroelectrics, etc.). Scattering of heat waves in a glass is commonly attributed to specific structural defects and the defect - induced additional low frequency local vibrational modes [1] which scatter acoustic modes. At present, the nature of quasilocal vibrational modes is being discussed to clarify their role in heat transport under the conditions of heavy resonance scattering of sound modes when acoustic and quasilocal low-frequency vibrational modes are actually interdependent excitations. The hybridization of phonons and local vibrational modes in the Ioffe-Regel crossover for inelastic scattering determines both the behavior of thermal conductivity and the other thermal and dynamic properties of glasses [2-5].

Two types of disorder can occur in molecular glasses: full structural disorder (both translational and orientational order are absent, glass) and orientational disorder (there is translational order of the molecular centers of mass, which form a crystalline lattice although molecular orientations are at random, usually known as orientational glass (OG) or orientationally - disordered crystal). The orientational degrees of freedom are of paramount importance to the dynamics of both molecular glasses and orientational crystals. The glass transition in ethyl alcohol occurs at $T_{g}=97 \mathrm{~K}$. The glass state is formed on fast cooling the liquid below $T_{g}$. The orientational glass appears on cooling a plastic crystal below the temperature of the transition to the orientationally-disordered phase. For ethyl alcohol this temperature practically coincides with $T_{g}$.

Deuteration has no effect on the main temperature fixed points for this substance, even though the molar mass of completely deuterated ethyl alcohol $\mathrm{C}_{2} \mathrm{D}_{5} \mathrm{OD}(52.11 \mathrm{~g} / \mathrm{mole})$ 
is about $14 \%$ larger than the molar mass of protonated ethyl alcohol $\mathrm{C}_{2} \mathrm{H}_{5} \mathrm{OH}$. Deuterated and protonated ethyl alcohols melt at about the same temperatures $\left(T_{m}=159 \mathrm{~K}\right)$ and the same applies to that signaling the transitions into the glass and orientationally-disordered phase. Both $\mathrm{C}_{2} \mathrm{D}_{5} \mathrm{OD}$ and $\mathrm{C}_{2} \mathrm{H}_{5} \mathrm{OH}$ can be considered as network solids due to the action of $\mathrm{H}$-bonds which are responsible for the chain-like structure in the condensed state. Hydrogen bonds afford a diversity of metastable states in the alcohols. Completely - deuterated and protonated solid ethanols are characterized by rich a polymorphism. Under equilibrium vapor pressure it can have three metastable long-lived phases and single stable thermodynamically equilibrium phase. The latter is an orientationally - ordered monoclinic crystal. Glass, orientational glass and a crystal with a dynamic orientational disorder (plastic crystal) are metastable but long-lived phases of ethanol.

The phases of deuterated ethyl alcohols can be obtained by the same technique as for the protonated alcohol [6]. Since the structural and orientational glasses of the deuterated alcohol exist in a wide range of temperatures, it is quite easy to compare the behavior of the thermal conductivity in theses phases within the same temperature interval.

Previously, the following features were detected in the thermal conductivity of some simple alcohols.

1. At high temperatures the thermal conductivity of orientationally-ordered crystals departs from the $1 / T$ law, which is attributed to the effect of libration vibrations of molecules. The strength of such a departure depends upon the mass of the alcohol molecule [7].

2. The curves $\kappa(T)$ measured on the orientational and structural glasses of ethanol [6] are similar in shape but the thermal conductivity of the orientational glass is slightly higher in magnitude. This suggests that the molecular orientational disorder is the main source of acoustic phonon scattering in the glass.

3. An anomalous thermal conductivity was revealed in the glass state and a glassy crystal at high temperatures [8].

The thermal conductivity of alcohol glasses $[6,9,10]$ was described by the soft potential model (SPM), which was first proposed by Karpov, Klinger and Ignatiev in 1983 to explain the universal properties of various glasses [11] and developed later in [12-17]. The model provides a unified description of thermal conductivities of various glasses in a wide range of temperatures.

Our purpose here is to find out how the thermal conductivity of ethyl alcohol in different solid phases changes as a result of complete deuteration. The substitution of deuterium for the hydrogen atoms in the ethanol molecule has only a slight effect on the translational subsystem. However, deuteration changes considerably the moment of inertia of the molecule $\left(I_{\mathrm{D}} / I_{\mathrm{H}}=1.23[18]\right)$, which is attended with changes in the libration spectrum. These spectral changes influence the heat capacity [18] and are expected to affect the thermal conductivity because they have effect on the phonon scattering by rotational excitations. In this study the thermal conductivity $\kappa(T)$ of fully deuterated alcohol was investigated in the phases of an orientationally ordered crystal $\left(T=2 \mathrm{~K}-T_{m}\right)$, an orientationally - disordered crystal and in the glass state $\left(T=2 \mathrm{~K}-T_{g}\right)$ at equilibrium vapor pressure. The results obtained were analyzed and compared with the previously obtained thermal conductivities of protonated ethyl alcohol.

\section{Experiment and discussion}

The thermal conductivity of different phases of solid $\mathrm{C}_{2} \mathrm{D}_{5} \mathrm{OD}$ was measured under equilibrium vapor pressure by means of a setup [19] using the steady-state potentiometric method. The container for the sample [19] was a stainless steel tube. Two copper wires $1 \mathrm{~mm}$ in diameter were passed through the container perpendicular to its axis, which permitted measurement of the average temperature along the isothermal plane running across the sample. At the outer surface of the container copper sockets were soldered to the wires to cartridge two temperature sensors. The liquid $\mathrm{C}_{2} \mathrm{D}_{5} \mathrm{OD}$ sample was put in the container of the measuring cell under $\mathrm{He}_{4}$ gas flow. The helium gas was used to improve the heat exchange between the sample and the container. The container with the sample was vacuumtight covered with the copper cap and an indium ring. A heater was fixed on the container cap to generate a downward heat flow in the sample.

Sample preparation and thermal conductivity measurements were completely automated including recording the thermal history parameters such as the temperatures registered by the upper and lower thermometers, the power of the sample heater, the current time, etc.

The deuterated $\mathrm{C}_{2} \mathrm{D}_{5} \mathrm{OD}$ alcohol (D purity was $99 \%$, anhydrous) was supplied by Cambridge Isotope Laboratories, Inc.

The samples in the glass and crystalline phases were prepared using the techniques applied to ethanol [6]. The different phases were prepared within the container using different cooling - heating cycles for the same sample and taking into account the thermal history. The glass was prepared by very fast cooling (above $50 \mathrm{~K} \mathrm{~min}^{-1}$ ) of the room-temperature liquids through their glass transition regions to the boiling temperature of liquid $\mathrm{N}_{2}$. Since the glass transition temperature $T_{g}$ of $\mathrm{C}_{2} \mathrm{D}_{5} \mathrm{OD}$ is higher than the boiling point of nitrogen, the glass sample was prepared by immersing the container with the sample directly into liquid nitrogen. The measurements of glass were performed at gradually decreasing temperature. After reaching the lowest point of the experiment the measurement was continued at increasing temperature. Above $T_{g}$ the glass sample transforms into a supercooled liquid.

A sample of orientational glass was prepared by cooling a plastic crystal. A liquid with initial room temperature was cooled fast enough $\left(\approx 1.5 \mathrm{~K} \mathrm{~min}^{-1}\right)$ to avoid crystalli- 
zation of the orientationally-ordered phase and to obtain a plastic crystal. The plastic crystal was cooled further at the same rate and on passing through the temperature of the orientationally - disordered transition (coincides with $T_{g}$ for $\mathrm{C}_{2} \mathrm{D}_{5} \mathrm{OD}$ ) it transformed into an orientational glass (OG) of deuterated alcohol. The thermal conductivity of the sample was measures on cooling to helium temperatures and then on heating. The absence of a hysteresis in the thermal conductivity indicated that the phase transitions were completed. The moments of the liquid-plastic crystal and plastic crystal - orientational glass phase transitions were seen as kinks in the curves of the thermogram of sample preparation.

A completely ordered crystal was obtained by heating an OG sample $\left(\approx 0.49 \mathrm{~K} \mathrm{~min}^{-1}\right)$, which results in spontaneous violent crystallization at $T \approx 148 \mathrm{~K}$ (seen as a peak in the thermogram). The sample was then annealed near $T_{m}$ for 24 hours and the thermal conductivity was measured first at decreasing and then at increasing temperatures. After measuring the thermal conductivity of the crystal, we tried to achieve slow crystallization and were thus able to obtain three more crystalline samples by the same procedure (crystal melting - cooling to obtain OG-OG heating). Crystallization was however violent and spontaneous at $T \approx 127 \mathrm{~K}, \approx 119 \mathrm{~K}$ and $\approx 115 \mathrm{~K}$. The thermal conductivities $\kappa(T)$ of the four crystal-phase samples coincided within the experimental error and are therefore indicated with the same symbols in Fig. 1 to avoid its overloading.

The thermal conductivity of the deuterated alcohol is shown in Fig. 1 for crystalline, glassy and orientationallydisordered crystalline (OG) phases. In the completely ordered crystal phase the thermal conductivity is described by a bell-shaped curve with a distinct phonon maximum at $T=14.2 \mathrm{~K}$. Below $T_{\max }^{\mathrm{ph}}$ the thermal conductivity is close to the $T^{2}$-dependence. Above $T_{\max }^{\mathrm{ph}}$ the thermal conductivity decreases exponentially changing to the $T^{-1}$-depen-

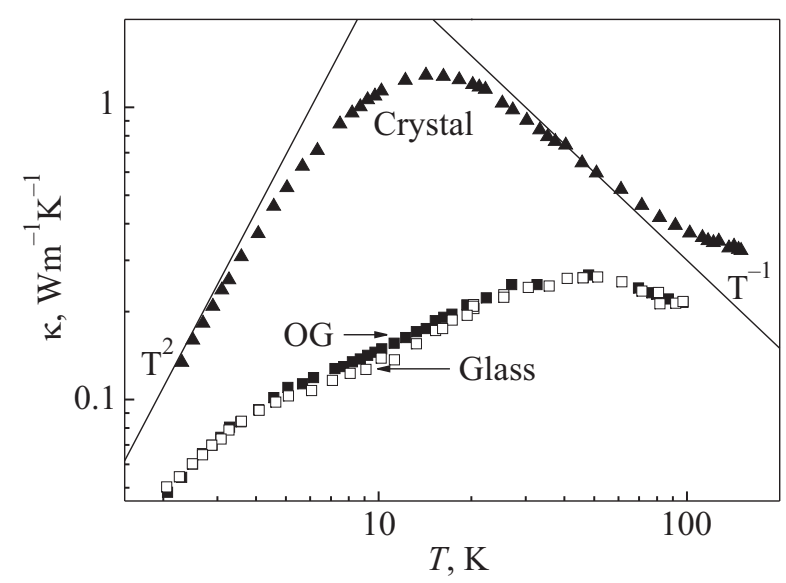

Fig. 1. The temperature dependence of the thermal conductivity of deuterated ethanol in the glass state $(\square)$, an orientationally-disordered crystal $(\mathrm{OG})(\boldsymbol{\square})$ and in a completely ordered crystal $(\boldsymbol{\Delta})$. dence. At $T>40 \mathrm{~K}$ there is an evident departure from the $T^{-1}$-dependence, like in simple alcohols (methanol, ethanol, 1-propanol) [7]. The thermal conductivity of the deuterated alcohol is much lower in the structural glass state and the OG phase than in a crystal and its behavior is close to that of glasses: $\kappa(T)$ grows with temperature, reaches a smeared plateau at $T \approx 10 \mathrm{~K}$ then increases up to a smeared maximum at $\approx 48 \mathrm{~K}$ and finally decreases as the temperature rises. The thermal conductivities of the glass state and OG phase are very close but their behavior differs slightly: in the position glass the curve $\kappa(T)$ has a little inflection in the region 5-20 K, the discrepancy between the curves being maximum at $10-15 \mathrm{~K}$.

Figure 2 illustrates the temperature dependences of the thermal conductivity measured on the equilibrium phase of a completely ordered crystal of deuterated ethanol. For comparison, the figure also carries the data for protonated ethanol [7]. The behavior of the curves is closely similar: as the temperature rises, the thermal conductivity increases by the law $\sim T^{2}$ and passes through a phonon maximum. In contrast to deuterated ethanol, the maximum thermal conductivity of the protonated alcohol occurs at $11 \mathrm{~K}$ and is 1.9 times higher. This indicates that despite prolonged annealing, crystals of deuterated ethanol are far more defective than those of hydrogenated ethanol. It is likely that deuteration enhances the crystal rigidity and makes it harder to remove defects even on annealing (like in pure crystalline hydrogen and deuterium [20]). This is related to the mobility of molecules: the molecules of deuterated ethanol have a larger mass and hence lower mobility in comparison with the $\mathrm{C}_{2} \mathrm{H}_{5} \mathrm{OH}$ molecule. As the temperature rises further, the thermal conductivities of both alcohols decrease exponentially and become equal at $T \approx 25 \mathrm{~K}$. In the premelting region the thermal conductivity of deuterated ethanol is about 1.2 times higher than that of the protonated alcohol. This is consistent with the previous conclusion [7]

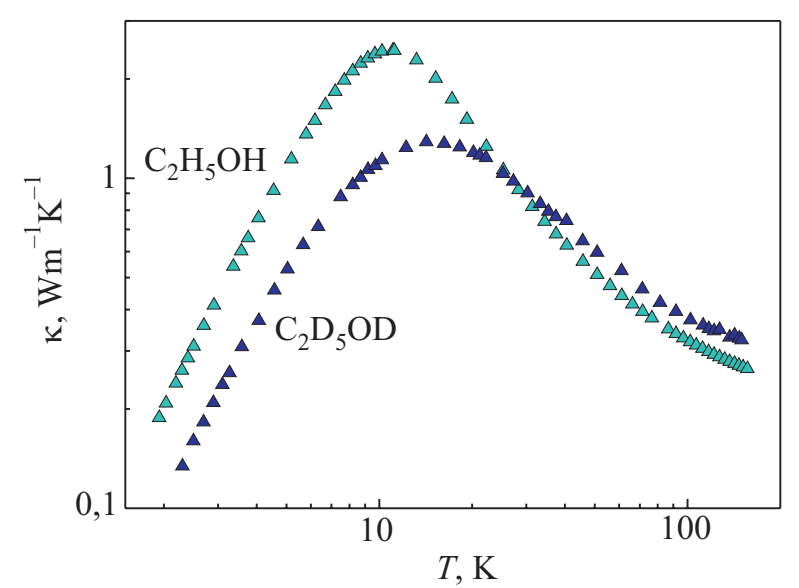

Fig. 2. The temperature dependences of the thermal conductivity of protonated [7] and deuterated ethanol in the crystal phase. 
about the dependence of thermal conductivity on the molecular mass of primary monohydric alcohols. Such dependence was observed in the orientationally - ordered phase: the larger was the molecular mass, the higher was the thermal conductivity in the premelting region.

The temperature dependences of thermal conductivity measured in the metastable phases (glass and OG) of the deuterated alcohol are shown in Fig. 3 in the semilogarithmic coordinates along with the corresponding data (the same phases) for protonated alcohol [6]. The thermal conductivities of both alcohols have much in common: their magnitudes practically coincide at low temperatures. The tendency of increasing magnitudes of thermal conductivity with an increasing mass of an alcohol molecule, which was detected in [21], also persists in the low temperature region. As the temperature rises, $\kappa(T)$ increases and passes through a maximum, which is at $T \approx 48 \mathrm{~K}$ in the deuterated alcohol and at $T \approx 51 \mathrm{~K}$ in the protonated one. On a further growth of the temperature the thermal conductivities of the alcohol decrease slightly. This decrease is anomalous, like in the case of [8], because the curve $\kappa(T)$ is monotonic for most glasses. The thermal conductivity in the OG phase of deuterated and protonated alcohols is slightly higher in the whole interval of temperature than in the glass state.

However, there are some distinctions between the thermal conductivities of the metastable phases. The curve $\kappa(T)$ has a distinct inflection at $5-20 \mathrm{~K}$ in the glass state of the deuterated alcohol. This feature is absent in the OG phase. As the temperature rises, the distinction between the curves $\kappa(T)$ of $\mathrm{C}_{2} \mathrm{D}_{5} \mathrm{OD}$ and $\mathrm{C}_{2} \mathrm{H}_{5} \mathrm{OH}$ increase: the maximum $\kappa(T)$ in the metastable phases (glass, OG) of deuterated ethanol are about 1.4 times higher in comparison with the protonated alcohol. Another interesting feature is that the difference between the thermal conductivities of the OG and glass phases is much smaller in the deuterated alcohol.

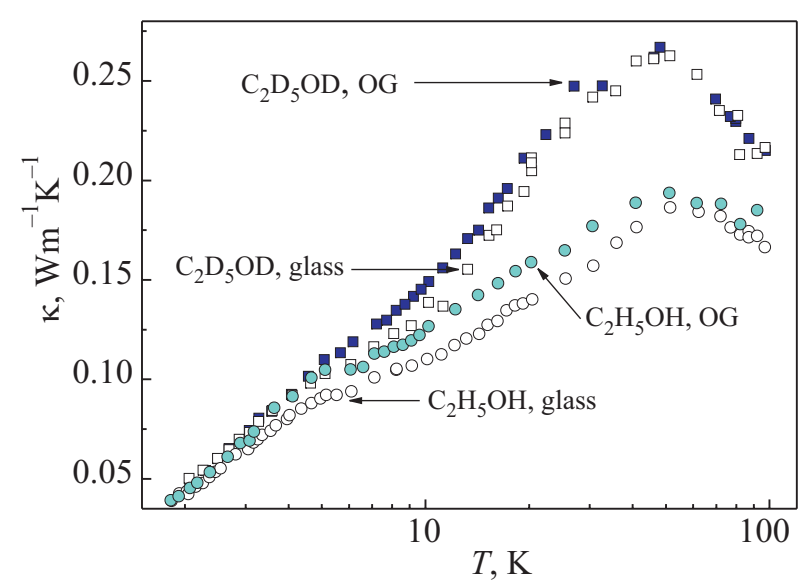

Fig. 3. The temperature dependence of the thermal conductivity of protonated (circles) and deuterated (squares) ethanols in the state of glass (open symbols) and in the phase of an orientationally-disordered crystal (closed symbols).

\section{Interpretation of experimental results}

The temperature dependence of the thermal conductivity in the solid phases of deuterated alcohol is rationalized assuming that it is given by a sum of two contributions [22-29]:

$$
\kappa(T)=\kappa_{\mathrm{I}}(T)+\kappa_{\mathrm{II}}(T) .
$$

The contribution $\kappa_{\mathrm{I}}(T)$ is made by the propagating phonons whose mean free path is larger than the phonon halfwavelength and are the main heat carriers at low temperatures. The contribution $\kappa_{\mathrm{II}}(T)$ is due to localized shortwavelength vibrational modes, or phonons whose mean free path is equal to the phonon half-wavelength. Above $40 \mathrm{~K}$ the thermal conductivity of a deuterated alcohol crystal can be approximated by the expression $\kappa(T)=A / T+B$, where $A / T$ describes phonon-phonon scattering (three-phonon Umklapp processes) and $B$ is an additional mechanism operating in the high-temperature region $\left(A=20.1 \mathrm{Wm}^{-1}\right.$, $B=(0.18 \pm 0.01) \mathrm{Wm}^{-1} \mathrm{~K}^{-1}$; for the protonated alcohol $A=16.9 \mathrm{Wm}^{-1}, B=(0.16 \pm 0.01) \mathrm{Wm}^{-1} \mathrm{~K}^{-1}$ [7]). This means that the parameter $A$ increases with increasing molecular mass (the strength of phonon scattering decreases), which agrees with previous findings [7]. In contrast, the parameter $B$ shows a far milder dependence with mass.

The Eq. (1) was previously employed to interpret the thermal conductivity of crystalline methanol, ethanol and 1-propanol [7] and glassy methanol [10]. In case of glasses the contribution $\kappa_{\mathrm{I}}(T)$ is well described in the soft potential model $[1,14,17]$, which describes the phonon scattering caused mainly by the low-energy excitations of a strongly anharmonic ensemble of particles. The dimensionless parameter $\bar{C}$ of the coupling between the phonon and two-level systems and the characteristic crossover energy $W$ are the most important parameters of the model. The energy $W$ is the boundary energy below which the phonons are scattered at the tunnel levels of a two-level system. Above this energy the phonons are scattered at soft quasiharmonic vibrations. The model is an efficient tool of describing the thermal conductivity of most glasses.

The temperature dependence of the thermal conductivity of the molecular glasses is determined by the standard expression derived within the Debye approximation for the density of states of acoustic modes:

$$
\kappa_{I}(T)=\frac{k_{B}^{4} T^{3}}{2 \pi^{2} \hbar^{3} v} \int_{0}^{\infty} d x \tau(x) \frac{x^{4} \mathrm{e}^{x}}{\left(1-\mathrm{e}^{x}\right)^{2}},
$$

Where $x=\left(\hbar \omega / k_{B} T\right), \omega$ is the phonon frequency, $v$ is the polarization - averaged sound velocity, $\tau(x)$ is the effective relaxation time of phonon scattering. In the soft potential model the inverse relaxation time $\tau^{-1}(x)$ can be subdivided into three components which describe acoustic wave scattering by the tunnel states $t_{t s}^{-1}$, by classical relaxation systems $t_{c r}^{-1}$ and by quasi-local vibrations $t_{l v}^{-1}$ : 


$$
\begin{gathered}
\tau^{-1}(\omega)=\tau_{t s}^{-1}+\tau_{c r}^{-1}+\tau_{l v}^{-1}= \\
=\bar{C} \pi \omega\left[1.1 \tanh \left(\frac{\hbar \omega}{2 k_{B} T}\right)+\left(\frac{T}{W}\right)^{3 / 4} \times\right. \\
\left.\times \ln ^{-1 / 4}\left(\frac{1}{\omega \tau_{0}}\right)+\frac{1}{6 \sqrt{2}}\left(\frac{\hbar \omega}{W}\right)^{3}\right],
\end{gathered}
$$

where $\bar{C}$ is a dimensionless constant characterizing the strength of coupling between a sound wave and the soft localized mode without regard for the difference between longitudinal and transverse sound modes. $W$ is the characteristic energy of the quartic potential of the SPM, and $\tau_{0}$ is an attempt frequency of the order of $10^{-13} \mathrm{~s}$.

Inserting Eq. (3) in Eq. (2), replacing $\omega$ by its dependence of $x$ and defining a dimensionless variable $z=\left(k_{B} T / W\right)$ (normalized temperature),

$$
\kappa_{I}(T)=\frac{2 k_{B}}{\pi}\left(\frac{W}{h}\right)^{2} \frac{1}{\bar{C} v} F(z),
$$

where

$$
F(z)=\int_{0}^{\infty} d x \frac{x^{3} \mathrm{e}^{-x}}{\left(1-\mathrm{e}^{-x}\right)^{2}} \frac{z^{2}}{1.1 \tanh (x / 2)+0.7 z^{3 / 4}+x^{3} z^{3} / 8} .
$$

The function $F(z)$ depends on the normalized (scaling by $W$ ) temperature $(z)$. Equation (4) is obtained from the product $\bar{C} v=C_{l} v_{l}=C_{t} v_{t}$ ( $v_{j}$ being the velocities of the longitudinal $(j=l)$ and transverse $(j=t)$ sound waves, $C_{l}$, $C_{t}$ are the corresponding coupling parameters) [16]. The temperature dependence $\kappa(T)$ is determined by the function $F\left(k_{B} T / W\right)$ and its magnitude is dictated by the combination of the parameters $W^{2} / \bar{C} v$. According to the SPM, the dependence $\kappa(T)$ is universal for glasses because the relation $\kappa(T)\left(\bar{C} v / W^{2}\right)$ as a function of $k_{B} T / W$ is independent on a particular structure and chemical composition of the substance at temperatures $k_{B} T / W \leq 2$.

The Fig. 4 illustrates the experimental results for deuterated and protonated [6] ethanol in the glass state and in the OG phase. The data are drawn in reduced coordinates:

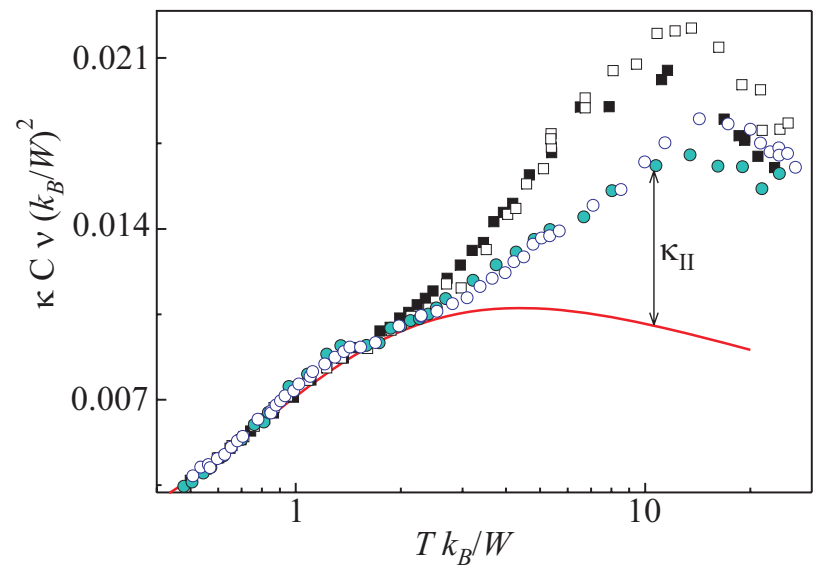

Fig. 4. The temperature dependence of the thermal conductivity of protonated (circles) and deuterated (squares) ethanols in the glass state (open symbols) and in the OG phase (closed symbols) in the reduced coordinates. Line is for theoretical SPM calculation. Arrow marks the contribution $\kappa_{\mathrm{II}}$ to the thermal conductivity of protonated ethanol in the OG phase for $T k_{B} / W=10$.

normalized temperature $T k_{B} / W$ on the abscissa, the product $\kappa(T) \cdot \bar{C} v\left(k_{B} / W\right)^{2}$ on the ordinate and the SPM - calculated universal curve. The experimental data coincide with the calculate curve at $T \leq 2 W / k_{B}$ and deviate from it at $T \geq 2 W / k_{B}$. The contribution $\kappa_{\mathrm{II}}$ is found as a difference between the experimental data and the calculated curve (the arrow marks $\kappa_{\mathrm{II}}$ for $T k_{B} / W=10$ of protonated ethanol in the OG phase).

The Table 1 gives the molar mass, Debye temperature, sound velocity, SPM parameters and the parameters of the model of [4] obtained through fitting the theoretical curve and the experimental results for protonated and deuterated ethanol in the glass state and the OG phase.

The sound velocities $v$ were calculated from the Debye temperatures (column 3) that had been obtained from the specific heat data for glasses $[18,30]$. Notice that the calculated $\Theta$ is $20-30 \%$ higher for crystals than for glasses from the specific heat data $[18,30]$, being $\Theta=136 \mathrm{~K}$ for protonated and $\Theta=129 \mathrm{~K}$ for deuterated alcohols. The parameter $\bar{C}$ changes only slightly. The parameter $W$ is higher in deuterated ethanol.

Table 1. Molar mass $M$, Debye temperature $\Theta$, sound velocity $v$, SPM parameters $\bar{C}, W / k_{B}$ and the parameters $\Theta_{u p}, \Theta_{\text {low }}, G$ of the model of [4] estimated from fitting calculated curves and experimental data for protonated and deuterated ethanol in metastable phases.

\begin{tabular}{|c|c|c|c|c|c|c|c|c|}
\hline \hline Alcohol & $M, \mathrm{~g} / \mathrm{mole}$ & $\Theta, \mathrm{K}$ & $v, \mathrm{~m} / \mathrm{s}$ & $W / k_{B}, \mathrm{~K}$ & $\bar{C}$ & $\Theta_{\text {low }}, \mathrm{K}$ & $G$ & $\Theta_{u p}, \mathrm{~K}$ \\
\hline \hline $\mathrm{C}_{2} \mathrm{H}_{5} \mathrm{OH}$, glass & 46.07 & 108 & 1514 & 3.6 & 0.00085 & $49 \pm 2$ & 1 & $94 \pm 2$ \\
$\mathrm{C}_{2} \mathrm{H}_{5} \mathrm{OH}, \mathrm{OG}$ & 46.07 & 110 & 1548 & 3.8 & 0.00082 & $45 \pm 2$ & 1 & $84 \pm 2$ \\
$\mathrm{C}_{2} \mathrm{D}_{5} \mathrm{OD}$, glass & 52.11 & 103 & 1440 & 3.8 & 0.00084 & $47 \pm 2$ & 2 & $80 \pm 2$ \\
$\mathrm{C}_{2} \mathrm{D}_{5} \mathrm{OD}, \mathrm{OG}$ & 52.11 & 104 & 1462 & 4.1 & 0.00090 & $46 \pm 2$ & 2 & $75 \pm 2$ \\
\hline \hline
\end{tabular}

The contribution $\kappa_{\mathrm{II}}(T)$ was obtained by subtracting $\kappa_{\mathrm{I}}(T)$ from the total thermal conductivity $\kappa(T)$. The temperature dependences of $\kappa_{\mathrm{II}}(T)$ are shown in Fig. 5. It is seen that in the glass state and in the OG phase the contributions $\kappa_{\mathrm{II}}(T)$ of two modifications of ethanol are closely similar. In deuterated ethanol $\kappa_{\mathrm{II}}(T)$ increases with temper- 


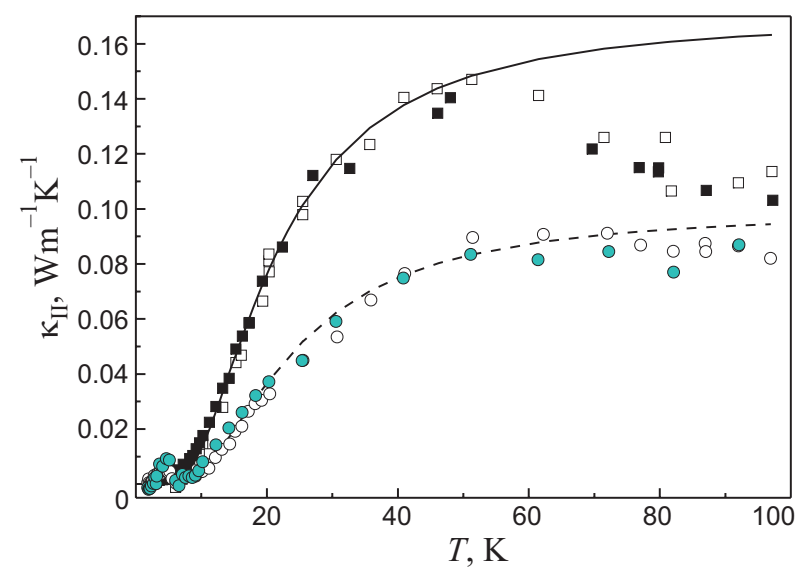

Fig. 5. The temperature dependence of $\kappa_{\text {II }}$ for protonated (circles) and deuterated (squares) ethanols in the glass state (open symbols) and the OG phase (closed symbols). Experimental data symbols, calculation by the Klinger model [4] - lines.

ature up to $50 \mathrm{~K}$. At $T=50 \mathrm{~K} \kappa_{\mathrm{II}}$ is $60 \%$ higher than $\kappa_{\mathrm{II}}$ of protonated ethanol. Then it decreases and at temperatures close to $T_{g} \kappa_{\mathrm{II}}(T)$ - values are close for two alcohol modifications. The contribution $\kappa_{\mathrm{II}}(T)$ describes the heat transfer by localized harmonic short-wavelength vibrational modes, or acoustic phonons whose mean free path is equal to the phonon half-wavelength. The simplest description of $\kappa_{\mathrm{II}}(T)$ is offered by the phenomenological Cahill-Pohl model [31] for interpretation the thermal conductivity of amorphous solids at high temperatures. The model assumes in the approximation of the Debye phonon spectrum for the isotropic medium (the difference in the phonon mode polarization is disregarded) that the minimum lifetime of each vibration is equal to its half-period time. Later on the Cahill-Pohl model was developed by Klinger and Halpern [4], who constructed a theoretical model of soft mode dynamics to describe vibrational terahertz anomalies for glasses containing both the Boson peak with the following "high-frequency sound". The glass is modeled as an isotropic elastic medium for acoustic phonons whose wavelength is much greater than the intermolecular distance. The medium contains randomly distributed soft modes with resonance extended harmonic vibrational excitations which interact with the phonons. The resulting excitations are described by the eigenstate spectrum which has two branches separated by a narrow spectral gap. The lower branch is to a greater extent acoustic while the upper one is of a mixed polarization obeying an acoustic-like dispersion law. It is identified as propagating sound-like waves up to the upper frequency limit $\omega_{\text {up }} \approx(2-5) \omega_{\mathrm{BP}}$, where $\omega_{\mathrm{BP}}$ is the frequency corresponding to the Boson peak energy $E_{\mathrm{BP}}$. We describe $\kappa_{\mathrm{II}}$ within the model of soft mode dynamics that is extended to the high frequency region [4]:

$$
\begin{aligned}
\kappa_{I I}(T)= & 3 G\left(\frac{\pi}{6}\right)^{1 / 3}\left(\frac{\rho N_{A}}{M}\right)^{2 / 3} k_{B} v\left(\frac{T}{\Theta_{\mathrm{up}}}\right)^{2} \times \\
& \times \int_{\Theta_{\mathrm{low}} / T}^{\Theta_{\mathrm{up}} / T} \frac{x^{3} \mathrm{e}^{x}}{\left(\mathrm{e}^{x}-1\right)^{2}} d x,
\end{aligned}
$$

where $\rho$ is the density, $M$ is the molar mass, $N_{A}$ is the Avogadro number, $v$ is the sound-like wave velocity (in our case it coincides with the mean sound velocity in Eq.(2)). $G$ is the dimensionless normalization parameter effectively allowing for the intensity of heat transfer by localized excitations including rotational ones, $\Theta_{\text {up }}$ and $\Theta_{\text {low }}$ are the temperatures corresponding to the upper and lower frequency limits for the propagating sound-like waves $\omega_{\text {up }}$ and $\omega_{\text {low }}$, respectively; $\omega_{\text {low }}>\omega_{\mathrm{BP}}$. The density of $\mathrm{C}_{2} \mathrm{H}_{5} \mathrm{OH}$ glasses was $\rho=958.2 \mathrm{~kg} / \mathrm{m}^{3}$ [9]. For $\mathrm{C}_{2} \mathrm{D}_{5} \mathrm{OD}$ glasses the density $\rho=1083 \mathrm{~kg} / \mathrm{m}^{3}$ was calculated from the molar volume data, the coefficient being the same as for protonated ethanol. The theoretical calculation within the Klinger model is shown in Fig.5 (lines). The model parameters $\Theta_{\text {up }}, \Theta_{\text {low }}$ and $G$ obtained from experimental data fitting are given in Table 1. The Klinger model provides a good description of the results in the whole temperature interval for protonated ethanol and only up to $T=50 \mathrm{~K}$ for the deuterated alcohol, i.e. the diffuse component of $\kappa_{I I}$ increases up to saturation as the temperature rises. To describe the results for the deuterated alcohol above $50 \mathrm{~K}$, the parameter $G$ was effectively reduced to $G=1$.

In the Klinger model the phonon spectrum was obtained for distinct propagating excitations. It is determined by hybridization of long-wavelength acoustic phonons and vibrational soft mode excitations. The hybridization enhances in the Ioffe-Regel crossover, while changing from weak inelastic phonon scattering relative to the soft mode excitations to strong scattering exactly in the vicinity of the spectral gap. The mean free path of the acoustic phonon becomes short in the vicinity of the gap at the frequency corresponding to the Ioffe-Regel crossover. The phonon mean free path $l$ in the metastable phases of deuterated and protonated ethanols was obtained from the measured thermal conductivity and the phonon heat capacity $c_{\text {ph }}$ calculated in the Debye model using the expression for thermal conductivity $\kappa(T)=c_{\mathrm{ph}} v l / 3$. The temperature dependence of the phonon mean free path is shown in Fig. 6 in the semilogarithmic coordinates for the glasses of protonated and deuterated ethanols (the corresponding data are closely similar for orientational glasses and are therefore not shown in the figure). The straight lines presented the Boson peak energies $\left(28.5 \mathrm{~K}\right.$ for $\mathrm{C}_{2} \mathrm{H}_{5} \mathrm{OH}$ and $30.2 \mathrm{~K}$ for $\mathrm{C}_{2} \mathrm{D}_{5} \mathrm{OD}$ ) recalculated in units of temperature. The Boson peak energy was found using the expression $E_{\mathrm{BP}}=0.75 W \bar{C}^{-1 / 3}$ and assuming that the phonon mean free path is equal to the phonon wavelength (Ioffe-Regel 


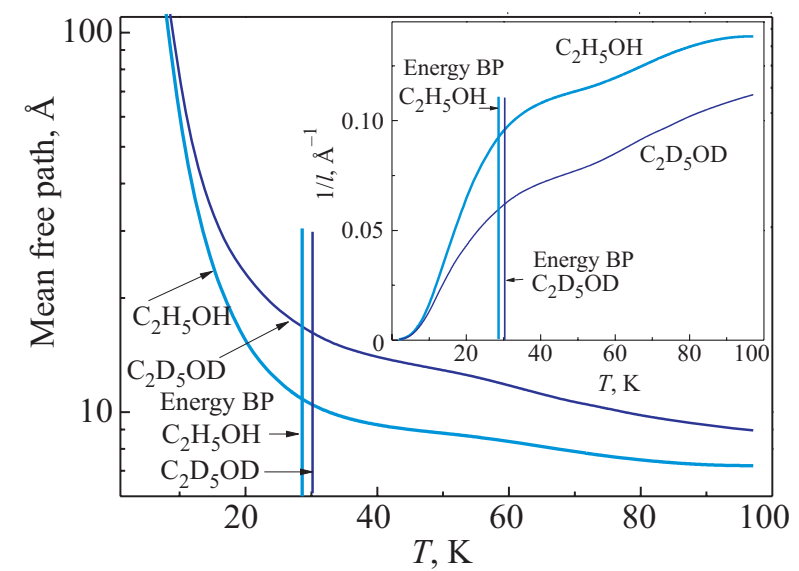

Fig. 6. The temperature dependence of the mean free path of phonons in the semilogarithmic coordinates for the glass state of protonated and deuterated ethanols. Insert: the temperature dependence of the inverse mean free path of phonons in the normal coordinates for glasses of protonated and deuterated ethanol. Straight lines show their Boson peak energies recalculated in the units of temperature, $T_{\mathrm{BP}}=E_{\mathrm{BP}} / k_{B}$, where $T_{\mathrm{BP}}$ is the Boson peak temperature.

crossover). It is seen in Fig. 6 that at low temperatures, $T<E_{\mathrm{BP}}$, the phonon mean free path is large for both alcohols. As the temperature rises, it decreases significantly and becomes short at $T>E_{\mathrm{BP}}$. The mechanism of phonon scattering changes in the region of the Boson peak energy. At $T>10 \mathrm{~K}$ the phonon mean free path $l$ increases appreciably in deuterated ethanol. Hence its thermal conductivity exceeds that of the protonated alcohol. In the SPM the inverse mean free path $1 / l$ is proportional to the phonon density of states. The insert in Fig. 6 shows the temperature dependence of $1 / l$ for glasses of deuterated and protonated ethanol in the normal coordinates. At $T>10 \mathrm{~K}$ the density of states increases faster in protonated ethanol. As a result, the phonon scattering in the protonated alcohol stronger than that for the fully deuterated sample and thus the thermal conductivity is lower.

\section{Conclusions}

The thermal conductivity of fully deuterated ethyl alcohol has been measured in the crystalline phase and two metastable phases (glass state and OG phase). In the ordered crystal deuteration strengthens the rigidity of the crystal (at medium and low temperatures the thermal conductivity of $\mathrm{C}_{2} \mathrm{D}_{5} \mathrm{OD}$ is lower than that of $\mathrm{C}_{2} \mathrm{H}_{5} \mathrm{OH}$ ). For the metastable phases (glass state, $\mathrm{OG}$ ) it is found that like in protonated ethanol [6] the molecular orientational disorder is the main source of acoustic phonon scattering in the glass state.

The trends exhibited for $\kappa(T)$ in the glass and OG of deuterated ethanol resemble those observed for the protonated material. In terms of its distinctive contributions the conductivity of both samples can be accounted for in terms of two contributions: $\kappa_{\mathrm{I}}(T)$ and $\kappa_{\mathrm{II}}(T)$, contribution ascribed to propagating phonons and short-wavelength vibrational modes, respectively. The effect of complete deuteration shows up in $\kappa_{\mathrm{II}}(T)$ predominantly. In both deuterated and protonated alcohols $\kappa_{\mathrm{II}}(T)$ is practically independent of the alcohol state (structural glass, orientationally-disordered crystal). This most likely reflects the decisive effect of the rotational degrees of freedom of the molecules on the heat transport in alcohols.

The authors are sincerely grateful to V.A. Konstantinov, V.G. Manzhelii, and M.A Strzhemechny for helpful and fruitful discussion and interest in this study.

1. U. Buchenau, Yu.M. Galperin, V.L. Gurevich, D.A. Parshin, M.A. Ramos, and H.R. Schober, Phys. Rev. B46, 2798 (1992).

2. I.A. Gospodarev, V.I. Grishaev, A.V. Kotlyar, K.V. Kravchenko, E.V. Manzhelii, E.S. Syrkin, and S.B. Feodos'ev, Fiz. Nizk. Temp. 34, 829 (2008) [Low Temp. Phys. 34, 655 (2008)].

3. Taras Bryk and Ihor Mryglod, Phys. Rev. B82, 174205 (2010).

4. M.I. Klinger and A.M. Kosevich, Phys. Lett. A280, 365 (2001), M.I. Klinger and A.M. Kosevich, Phys. Lett. A295, 311 (2002), M.I. Klinger and V. Halpern, Phys. Lett. A313, 448 (2003).

5. B. Rufflé, D.A. Parshin, E. Courtens, and R. Vacher, Phys. Rev. Lett. 100, 015501 (2008).

6. A.I. Krivchikov, A.N. Yushchenko, V.G. Manzhelii, O.A. Korolyuk, F.J. Bermejo, R. Fernandez-Perea, C. Cabrillo, and M.A. Gonzalez, Phys. Rev. B74, 060201 (2006); F.J. Bermejo, R. Fernandez-Perea, C. Cabrillo, A.I. Krivchikov, A.N. Yushchenko, O.A. Korolyuk, V.G. Manzhelii, M.A. Gonzalez, and M. Jimenez-Ruiz, Fiz. Nizk. Temp. 33, 790 (2007) [Low Temp. Phys. 33, 606 (2007)].

7. A.I. Krivchikov, F.J. Bermejo, I.V. Sharapova, O.A. Korolyuk, and O.O. Romantsova, Fiz. Nizk. Temp. 35, 1143 (2009) [Low Temp. Phys. 35, 891 (2009)].

8. AI. Krivchikov, A.N. Yushchenko, O.A. Korolyuk, F.J. Bermejo, C. Cabrilo, and M.A. Gonzalez, Phys. Rev. B75, 214204 (2007).

9. A.I. Krivchikov, A.N. Yushchenko, and O.A. Korolyuk, F.J. Bermejo, R. Fernandez-Perea, I. Bustinduy, and M.A. Gonzalez, Phys. Rev. B77, 024202 (2008).

10. O.A. Korolyuk, A.I. Krivchikov, I.V. Sharapova, and O.O. Romantsova, Fiz. Nizk. Temp. 35, 380 (2009) [Low Temp. Phys. 35, 290 (2009)]

11. V.G. Karpov, M.I. Klinger, and F.N. Ignatiev, Zh. Eksp. Teor. Fiz. 84, 761 (1983).

12. A. Il'in, V.G. Karpov, and D.A. Parshin, Sov. Phys. JETP 65, 165 (1987).

13. U. Buchenau, Yu.M. Galperin, V.I. Gurevich, and H.R. Schober, Phys. Rev. B43, 5039 (1991).

14. D.A. Parshin, Phys. Scr. 49A, 180 (1993). 
15. L. Gil, M.A. Ramos, A. Bringer, and U. Buchenau, Phys. Rev. Lett. 70, 182 (1993).

16. D.A. Parshin, Phys. Rev. B49, 9400 (1994).

17. A. Ramos and U. Buchenau, Phys. Rev. B55, 5749 (1997).

18. C. Talon, M.A. Ramos, and S. Vieira, Phys. Rev. B66, 012201 (2002); M.A. Ramos, C. Talon, R.J. JimenezRioboo, and S. Vieira, J. Phys.: Condens. Matter 15, S1007 (2003).

19. A.I. Krivchikov, V.G. Manzhelii, O.A. Korolyuk, B.Ya. Gorodilov, and O.O. Romantsova, Phys. Chem. Chem. Phys. 7, 728 (2005); A.I. Krivchikov, B.Ya. Gorodilov, and O.A. Korolyuk, Instrum. Exp. Tech. 48, 417 (2005).

20. O.A. Korolyuk, A. I. Krivchikov, and B.Ya. Gorodilov, JLTP 122, 203 (2001).

21. A.I. Krivchikov, O.A. Korolyuk, I.V. Sharapova, O.O. Romantsova, F.J. Bermejo, C. Cabrillo, R. Fernandez-Perea, and I. Bustinduy, J. Non-Crystalline Solids 357, 483 (2011).

22. V.A. Konstantinov, V.G. Manzhelii, M.A. Strzhemechny, S.A. Smirnov, Fiz. Nizk. Temp. 14, 90 (1988) [Low Temp. Phys. 14, 48 (1988)].

23. A. Jagannathan, R. Orbach, and O. Entin-Wohlman, Phys. Rev. B39, 13465 (1989).
24. H. Boettger and Th. Damker, Phys. Rev. B50, 12509 (1994); T. Damker, H. Böttger, and V.V. Bryksin, Phys. Rev. B59, 8626 (1999).

25. Philip B. Allen and Joseph L. Feldman, Phys. Rev. B48, 12581 (1993).

26. Joseph L. Feldman, Mark D. Kluge, Philip B. Allen, and Frederick Wooten, Phys. Rev. B48, 12589 (1993).

27. P.B. Allen, J.L. Feldman, J. Fabian, and F. Wooten, Philosophical Magazine B79, 1715 (1999).

28. A.J.H. McGaughey and M. Kaviany, Int. J. Heat and Mass Transfer 47, 1783 (2004).

29. Jerzy Bodzenta, Chaos, Solitons, \& Fractals 10, 2087 (1999).

30. C. Talon, M.A. Ramos, and S. Vieira, G.J. Cuello, F.J. Bermejo, A. Criado, M.L. Senent, S.M. Bennington, H.E. Fischer, and H. Schober, Phys. Rev. B58, 745 (1998).

31. D.G. Cahill and R.O. Pohl, Ann. Rev. Phys. Chem. 39, 1, 93 (1988); D.G. Cahill and R.O. Pohl, Phys. Rev. B35, 10, 4067 (1987); D.G. Cahill, S.K. Watson, R.O. Pohl, Phys. Rev. B46, 6131 (1992). 